MODELING, IDENTIFICATION AND CONTROL, 1990, VOL. 11, NO. 1, 49-54

doi:10.4173/mic.1990.14

\title{
Improvements in metallurgical processes by simulation
}

\begin{abstract}
KJELL O. SOLBERG†
Keywords: Metallurgy, simulation, process design, process control.

The metallurgical industry is very important to Norway. Traditionally cheap hydro-power has been the basis for this energy-intensive industry. This advantage is now gradually disappearing. To be compatible in the future, a superior technology has to be developed. For this purpose simulation has been taken into extensive use. Close cooperation is established between the industry and research institutes. This is vital for success. Simulation has proved to be an important tool in two areas: the optimum design of processes and the continuous supervision to keep them running at optimum. This paper gives examples of how substantial improvements have been obtained in the production of aluminium and ferro-alloys. Based on this experience the author draws some general conclusions on how to utilize simulation to increase industrial productivity. In the future a new area is envisaged; simulation in the development of advanced materials and products.
\end{abstract}

\section{Introduction}

By a small twist of an old saying I will state that 'Simulation is power'. As supposedly intelligent creatures we use our knowledge about the physical/chemical/mechanical world to shape it to our advantage. The design and operation of industrial processes are very complicated. We need efficient tools, and simulation models are very important. This is my main message.

When we want to manipulate a piece of equipment we activate our imagination, make some 'simulations' intelligently chosen among the vast number possible. Having arrived at a satisfactory or optimum performance, we go to the real world to implement the result. Note that the word 'imagination' contains 'image'. A simulator is also an image. Our mental simulation will however be too limited to treat complex industrial plants. We use the computer to assist us. Today not only the simulation per se is placed in the computing machine. Some of the reasoning based on experience, heuristics, is also being taken over by computers (AI or expert systems).

Before we go to specific examples let us analyse a bit more in detail exactly how simulations contribute to the claimed results. There are three areas; process design, process operation and product development. These are treated in the following.

\section{Process design}

This area can again be separated in three domains: simulation of laboratory experiments, of pilot plants, and of full scale industrial production.

Received 7 August 1989.

† Institute of Energy Technology, PO Box 40, 2007 Kjeller, Norway.

This paper was presented at SIMS '89 (Scandinavian Simulation Society), 31st Annual Meeting, Bergen, Norway, May 31-June 2, 1989. 


\subsection{Laboratory experiments}

Here simulation can be used to

select the critical parameters to be measured

design/optimization of the experiment to yield maximum information for minimum costs

interpret the experimental results, enhance basic understanding skip the laboratory work, save money, gain time

In all these areas we see that simulation contributes to the productivity of the often tedious, time consuming and costly laboratory experiment.

\subsection{Pilot plants}

Much the same as for laboratory experiments, simulation can be used to

design the optimum pilot plant

interpret the results

skip the whole costly and time (money) consuming pilot plant step

\subsection{Full industrial scale}

Here often sophisticated and very detailed simulations are needed to

design the plant

integrate/optimize with respect to energy and material use

minimize environmental pollution

assure that the integrated process is stable and controllable

design the control system

\section{Process operation}

Also here we distinguish between three application areas, process state measurements, fault diagnosis and predictive control.

\subsection{Measurement interpretation, process state determination}

On-line simulation models are used to

determine the important process states from secondary measured values, inverse modeling

filter out process and measurement noise

create redundancy by computed, simulated measurements allowing instrument checking etc.

coordination of various measurement signals into a consistent process state avoid costly measurements, replace by simpler and cheaper ones

All these features aim to increase the productivity of measuring.

\subsection{Fault diagnosis}

On-line process simulation can be used to:

detect as early as possible any faults so that preventive actions can be taken before the production is severely affected 
diagnose as precisely and quickly as possible the real cause for process malfunction. It is said that $80 \%$ of the time is used to find the error and $20 \%$ to repair it. It pays to reduce the $80 \%$

prevent the operator being confused and taking counterproductive steps by reducing to the essential the information presented to him

\subsection{Preventive control}

Simulation can be used to calculate the effects of various control actions so that the best one can be chosen before being included in the process. In all these cases of process operation the aim of the simulation is to minimize the negative effects of all kinds of disturbances. For the productivity of an industrial process it is extremely vital to keep the production undisturbed hopefully $100 \%$ of the time.

Emergency shutdowns are very costly and may also result in a bad reputation in the market. Time schedules are not kept. Such modern concepts as 'just in time' and 'custom tailored delivery' will put even more stress on continuous, smooth operation and rapid changes must be made to new orders without disruptions.

\section{Product development}

A fairly new area of simulation is in product design and development of advanced materials. The aim can be

analyse various shapes before the optimum is chosen (autos etc.)

choose appropriate materials to give the product optimum quality (paper, mechanical parts etc.)

select the right production steps (heat treatment, rolling, etc.) to minimize production costs without impeding the required quality

develop new materials by simulation of how the properties depend on a microstructure, alloying etc.

\section{Examples of improvements gained}

\subsection{Aluminium}

Aluminium is produced by the electrolysis of an oxide at $960^{\circ} \mathrm{C}$. The current is typically in the order of $200,000 \mathrm{~A}$, voltage $4.5 \mathrm{~V}$. This process has been subjected to extensive modeling.

The high current creates electromagnetic forces which will put the electrolyte into motion. It is important to arrange the electricity supply so that the cells do not disturb each other too much. Various geometries have been simulated, and a design has been found which allows the cells to be placed close together side by side. Thus the production per unit area has been increased.

The same problem is relevant to each cell. By extensive simulations of alternative designs it has been possible to increase the current per cell from $150,000 \mathrm{~A}$ to $240,000 \mathrm{~A}$, giving a proportional increase in production for the same capital investment.

The electrolysis cell design is a delicate balance between the shortest possible interpolar distance (minimum specific energy consumption) and the Al-yield. If too short a distance, $\mathrm{Al}$ will backreact and the current is not productive. This phenomenon is studied by combined analysis of the side freeze (thermal insulation), the paths of the current and how smoothly the electrolyte can be made to flow. Today the specific energy consumption is $25 \%$ less than when the simulation work was started. At the same time the productivity of the current has been increased by $12 \%$. 
The continuous control of the electrolysis process has improved vastly giving undisturbed production for a larger time with less energy consumption. The very few measurements made are interpreted by an online model, and this is used to control the material and energy balance. Previously the cells were run into a state called 'bluss' which precisely determined oxide concentration. This overvoltage is however very unfavourable, energy is wasted and violent disturbances are introduced.

Let us consider another part of the process: the production of carbon blocks used in the electrodes. These are produced in a separate factory where a given mixture of raw materials is baked in a very big oil-fired oven. Detailed 3D simulations of the temperature field as function of time were carried out, giving improvements in oven design and operation such that substantial energy savings were obtained.

We now move downstream to the casting of Al. This is done (semi)continuously in circular and rectangular shapes. For the quality (and price) of the product it is essential that the surface is completely smooth and that the crystal structure inside is as required (important for the following production steps). Extensive 3D simulation models have been developed both for the dynamic temperature field and the resulting stresses and strains. By using these the appropriate casting conditions can be chosen for each alloy. The quality of the products has been increased to be within narrow limits. Further, material waste due to fracture in the casting start-up has been eliminated.

The rolling process has also been modeled. A very effective way to produce Al-plates it to go directly from liquid $\mathrm{Al}$ to solid plates by water-cooled rolling. This process, as you can imagine, has to be very carefully controlled. The appropriate conditions are chosen based on model simulations.

\subsection{Steel/iron and ferro-alloys}

Ferro alloys are produced by reduction with coal/coke in large electric furnaces. One such machine typically needs $50 \mathrm{MW}$ (the consumption in a town of 10,000 inhabitants), and the power is supplied by 3 electrodes of up to $2 \mathrm{~m}$ diameter.

Complex simulators have been developed to study and optimize the oven design. These calculate the interconnected electric and magnetic fields. To describe the geometry we arrived at $3 \times 3 \mathrm{D}$ orthogonal coordinate systems generated by conformal mapping, having common boundary conditions at $120^{\circ}$ sector lines.

Separate and very detailed models have been developed and used for the design and operation of the electrodes. These are consumed and produced continuously. It is essential that they have sufficient mechanical strength when they enter the high temperature region. Various designs have been tested out with mathematical simulation.

At shut-down and start-up the electrodes sometimes can break. The problem was analysed by the electrode-simulator and turned out to be due to excessive thermal stresses. By simulation new start and stop procedures were determined such that electrode breaking was avoided. This is significant, one day off gives a loss of approximately 1 million Norwegian Kroner.

It is extremely important to detect any tendency to malfunction as early as possible. Countermeasures can be taken before any serious breakdown occurs. It is also a matter of personal safety. Tons of material at $1500^{\circ} \mathrm{C}$ must not escape from containment. For the purpose of process supervision an on-line diagnosis system has been developed. This makes use of real time simulation models to interpret the measurements. The following critical conditions are monitored; insulation in the bottom of the oven, cooling of the power supply system, cooling of the top lid, leakage of cooling water (can 
cause explosion), the supply of raw material, and the mechanical load on the three electrodes (the oven rotates). When this system is applied to all these furnaces in Norway, a gain of hundreds of MNOK per year is expected. This implementation is now underway.

Extensive simulation programs for steel working in product development have been produced. The simulation tool is sold under the name of STEELTEMP, in cooperation with MEFOS, Luleá, Sweden. STEELTEMP contains options for a variety of steel working: teeming, cooling, stripping, ingot heating, open die forging, flat rolling and groove rolling etc. The STEELTEMP program is being used by most of the steel works in Scandinavia.

These examples show that mathematical simulation is a tool of very high industrial value.

\section{Essential factors for success}

From my experience in metallurgical process simulation I would say the key parameters for success (when used for process improvement) are

1. The process engineer from the industry must play a strong role in the simulation development. Knowledge in the mathematics and technology of simulation alone is not sufficient. Experts claim that the simulation engineer preferably should be a process man who has acquired knowledge in simulation!

2. The problem should be clearly stated. This seems to be obvious, but nonetheless has to be stressed. Many academics are attracted by simulation as a toy to be played with. A golden rule is-do not model a process, make a model to solve a given problem.

3. Build a mental simulator before you make a mathematical one. The brain is very effective in analysing the broad scope of a problem, you first of all familiarize yourself with the real obstacles to problem solving. Next you avoid throwing away time and money on irrelevant work. The development of a simulator is expensive, it is important to use your brain before you start the programming.

4. Do some rough calculations to obtain a proper sense of scale. This step is important in two ways: it often results in simpler models as you are able to disregard unimportant features and you get a deeper understanding of the process.

5. Models and simulators must be verified by experimental data. This comparison may reveal some discrepancies either due to direct errors or incomplete description of the process at hand. If simulators are to be used with confidence, we must be sure they do agree with reality in some reference case.

6. The industrial partners must give sufficient priority to the simulation work. In addition to being heavily involved in the development, they must take over the simulator and use it as an integral tool in their work. This requires that the company has a sufficiently competent milieu in the modeling area. I think the real gain is a 'cultural lift' to the industry which affects its whole attitude towards advanced information technology.

\section{Thoughts about the future}

Process simulation will probably increase quite dramatically both in scope (areas of application) and in depth (accuracy of models). This is due to two facts, the development of the technology (software and hardware) and the need for the industry 
to be competitive. I believe that in high-cost countries the most important factor for productivity increases in process industries will be the ability to make use of simulation techniques for optimum design and operation.

I would like to emphasize a new area, simulation for product optimization. Our metallurgical industry will have to face higher quality demands and custom tailored products. New advanced materials and alloys open a whole, new field of product design. With modern simulation techniques it will be possible to predict the properties based upon choice of material and processing method.

The 'numerical process laboratory' may become a reality in the near future. In this laboratory time consuming and costly experimental work is to a large extent abandoned in favour of rapid and flexible computer simulations. Up to now simple correlations have been used to describe difficult phenomena. These will be replaced by simulation of the basic physical features giving a new dimension and fidelity to computation.

A major challenge to you is to bridge the gap between the academic, puristic domain and the real world of the industry. Let us show how the benefits can be reaped by high quality and goal-oriented work. 